\title{
Hypoxia-Induced Oxidative Stress in Health Disorders
}

\author{
Vincent Pialoux ${ }^{1}$ and Rémi Mounier ${ }^{2}$ \\ ${ }^{1}$ Centre de Recherche et d'Innovation sur le Sport, EA 647, Université Claude Bernard Lyon 1, 27-29 Rue du 11 Novembre 1918, \\ Campus La Doua, Villeurbanne, 69622 Villeurbanne, France \\ ${ }^{2}$ Équipe Génétique et Physiopathologie de Maladies Neurodéveloppementales et Neuromusculaires, Institut Cochin, \\ UMR8104 CNRS, INSERM U1016, Université Paris Descartes, 75014 Paris, France
}

Correspondence should be addressed to Vincent Pialoux, pialouxvincent@yahoo.fr

Received 27 November 2012; Accepted 27 November 2012

Copyright ( 2012 V. Pialoux and R. Mounier. This is an open access article distributed under the Creative Commons Attribution License, which permits unrestricted use, distribution, and reproduction in any medium, provided the original work is properly cited.

Chronic hypoxia has been shown to promote a large number of pathologies such as hypertension, cardiovascular and metabolic disorders, and respiratory diseases. The oxidative stress that occurs during intermittent or continuous cellular hypoxia is likely involved in all these diseases. Indeed, the reactive species were demonstrated to inhibit active substances, modulate the signaling of intracellular pathways, and mediate enzymes activation, which are known to play a critical role in the geneses and/or the outcomes of these pathologies.

The four review articles of this special issue describe the current knowledge regarding the role of oxidative stress in ischemic retinopathy, pulmonary diseases, and infertile testis and discuss a new model of yeast to study hypoxia-induced oxidative stress. In addition, the two original research articles presented in this issue further expand the understanding of the redox biology in the context of pulmonary arterial hypertension and impaired spermatogenesis induced by hypoxia.

The review article by S.-Y. Li et al. presents the underlying mechanisms involved in the hypoxia/ischemia-induced oxidative damage in diabetic retinopathy and retinopathy of prematurity. In particular, the authors have discussed the effect of therapeutic strategies of antioxidants treatment such as administration of catalase and superoxide dismutase, vitamin $\mathrm{E}$, and lutein and inhibition of NADPH oxidase or similar signaling pathways in these retinal ischemic diseases.

The extensive review by O. F. Araneda and M. Tuesta reports recent in vitro and in vivo experimental evidence that shows the implied mechanisms in pulmonary redox state by hypoxia via the increase of ROS generation in mitochondria, as from activation of NADPH oxidase, xanthine oxidase/reductase, and nitric oxide synthase enzymes, as well as throughout inflammatory process. Then, the role and impact of enzymatic and nonenzymatic antioxidant in the modulation of the pathways involved in the physiopathological response to hypoxia are extensively discussed. In a last part, the authors report the evolution of the two most studied makers of oxidative stress (exhaled nitric oxide for humans and pulmonary content of malondialdehydes for animals) in the lung in response to hypoxic exposure.

The paper of J. G. Reyes et al. synthesizes the current state of knowledge of the physiology of the testicles under pathologic hypoxic conditions that lead to reduced spermatogenesis. The molecular events triggered by all causes of hypoxia in the testis share common mechanistic pathways involving ROS generated by mitochondrial dysfunction and activation of enzymes such as xanthine oxidase or the inducible nitric oxide synthase. The recent results analyzed suggest that conditions such as germ cell apoptosis and DNA damage are common features in hypoxic testicles such as varicocele and testicular torsion. In addition, the oxidative damages present in hypoxia suggest the ROS play a role in the initiation stages of germ cell damage and apoptosis.

In another review by M. I. G. Siso and M. E. Cerdán the yeast Kluyveromyces lactis $\mathrm{K}$. is proposed as a respiratory eukaryote model, complementary to the fermentative $S$. cerevisiae, for the study of the pathways of hypoxia-induced oxidative stress. Although these two yeasts share homology between their acting proteins, the experimental studies 
described in this review reveal that there are many differences from a comparative perspective with respect to the signaling pathways and mechanisms of cells regulation including life span adjustment, programmed cell dead, autophagy, and mitophagy.

The paper by A. Zepeda et al. tests the protective and antioxidant effects of a blueberry extract in testis of rats exposed to hypobaric hypoxia. The authors demonstrated that the decreased apoptosis of the testicles cells observed with such supplementation was probably driven by a reduced lipid peroxidation and increased antioxidant enzymes activities. This suggests that blueberry extract may have a high antioxidant capacity and that its regular ingestion could prevent the testicles disorders related to oxidative stress induced by hypoxia.

Finally, M. Mata et al. address the relationship between the expression of the proliferator-activated receptor $\gamma$ coactivator- $1 \alpha$ (PGC- $1 \alpha)$, the pulmonary vascular resistance, and the oxidative stress (cytochrome $\mathrm{c}$ and SOD expression) in patients with pulmonary arterial hypertension. The results suggest that PGC- $1 \alpha$ likely modulates by oxidative mechanisms at the mitochondrial level of patients with pulmonary hypertension and could be used as a biomarker since it is not expressed healthy subjects.

In conclusion, the growing body of evidence suggests that the paradoxical increase in ROS generation is at the heart of the pathologic mechanisms associated with the hypoxic feature. In this respect, we are convinced that the study of the underlying mechanisms increasing oxidative stress in the pathologies associated with hypoxia will be essential to develop the future therapies/treatments of these diseases.

\section{Acknowledgments}

This special issue would not be possible without the great efforts of the authors and the reviewers. In this regard, we would like to thank all these people that took part in the achievement of this issue.

Vincent Pialoux Rémi Mounier 


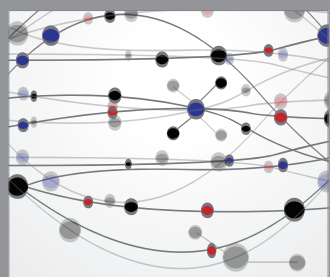

The Scientific World Journal


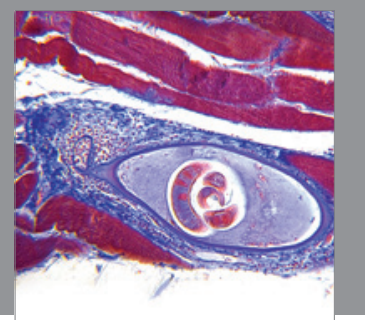

Gastroenterology

Research and Practice
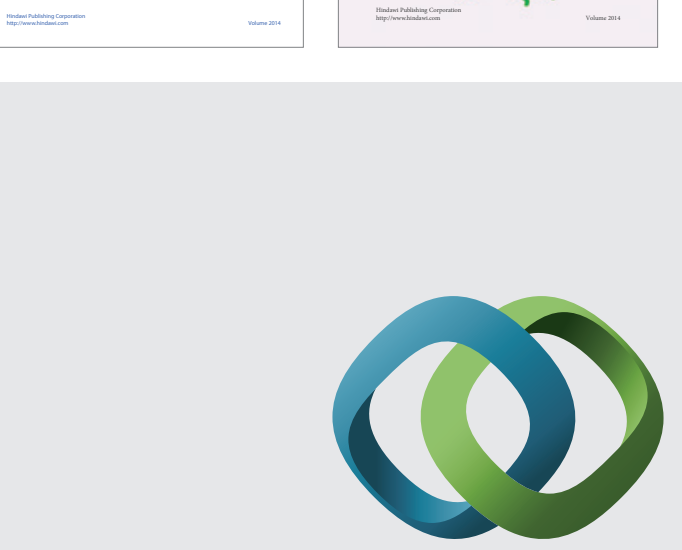

\section{Hindawi}

Submit your manuscripts at

http://www.hindawi.com
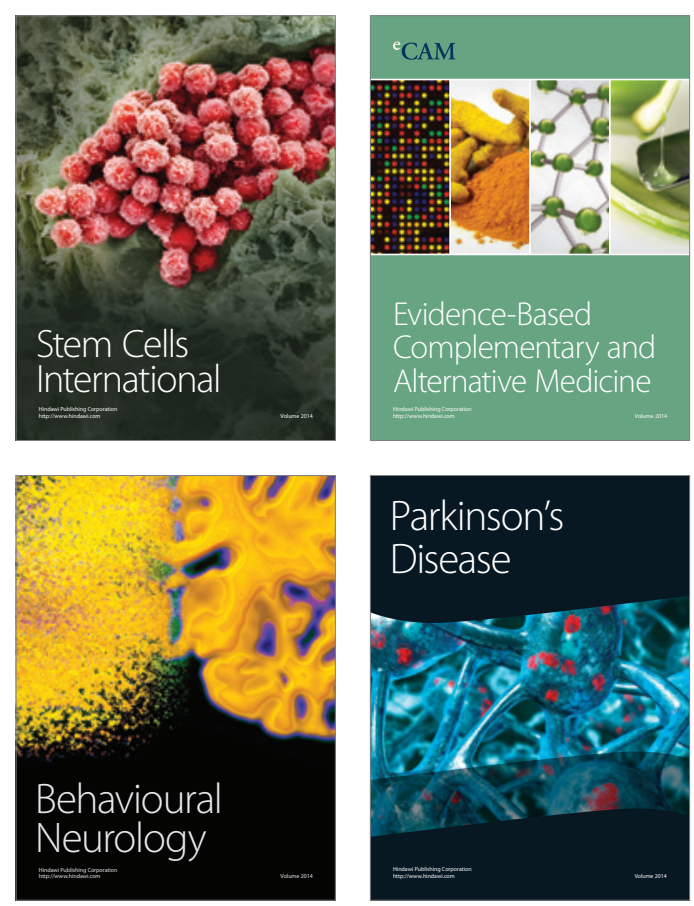

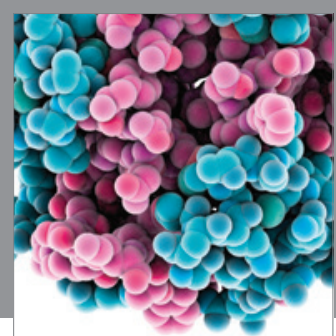

Journal of
Diabetes Research

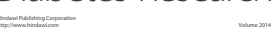

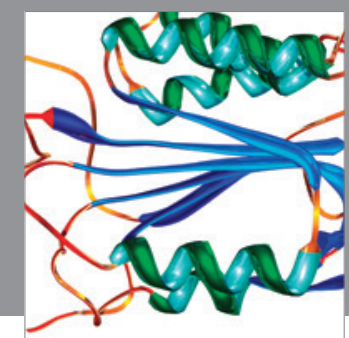

Disease Markers
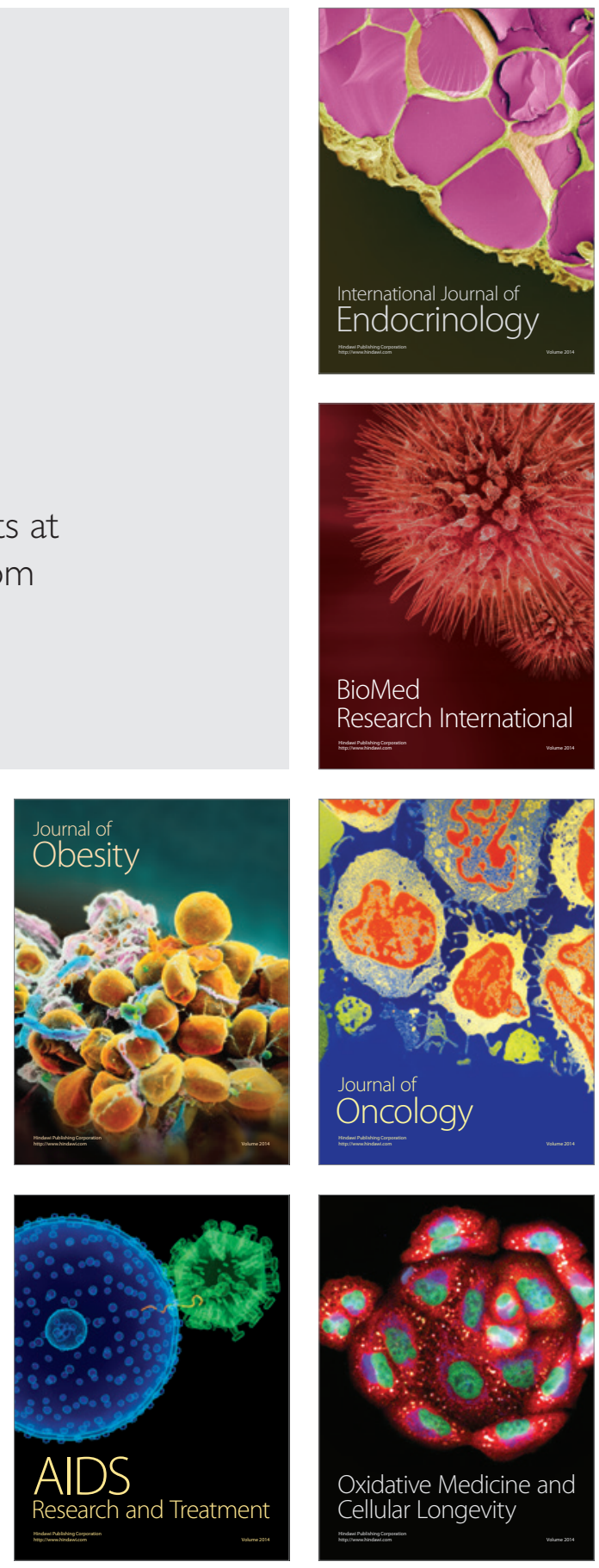